\title{
Modelling Covid-19 mitigation and control strategies in the presence of migration and vaccination: the case of South Africa
}

\author{
S. M. Kassa ${ }^{1}$ (D) J. B. H. Njagarah ${ }^{1}$ Y. A. Terefe ${ }^{2}$
}

Received: 16 October 2020 / Accepted: 30 April 2021 / Published online: 14 May 2021

(c) African Mathematical Union and Springer-Verlag GmbH Deutschland, ein Teil von Springer Nature 2021

\begin{abstract}
The novel coronavirus disease has ravaged many health systems around the world and has brought many economies to their knees. In the absence of an approved curing medicine or approved vaccine to date, the major control of the surge of infections is through use of NonPharmaceutical Interventions (NPIs) and imposing specific standard operating procedures (SOPs) in instances when the disease spread curbs are relaxed. It is thus essential to quantify the extent to which specific NPIs can be useful in containing the pandemic. To achieve this, we constructed a mathematical model that accounts for both person to person transmission as well as transmission through contact from pathogen-contaminated surfaces. The model assumes that there is change of behaviour resulting from the surge of the number of cases, hence a class of susceptible individuals who practise self-protection measures. Basic properties of the model including the conditions for existence and stability of steady states are explored. The model was fitted to new-cases data for South Africa and baseline parameter values were estimated. Sensitivity analysis of the model was performed to determine the most influential parameters on the disease threshold. Our results show that practising of selfprotection measures is vital in slowing the spread of the infection. In addition, it is evident from the results that minimizing contact through "physical distancing" as well as with contaminated surfaces can significantly help in containing the infection. The model was extended to account for testing and quarantining of both symptomatic and asymptomatic infected individuals. In addition, migration and potential use of a vaccine were explored. In the case of migration, the scenarios considered included aspects when there are both border control and illegal crossings as well as the case where the government is in full control with proper SOPs. Our results show that, although testing and isolating/quarantining of infected individuals is essential in
\end{abstract}

S. M. Kassa

kassas@biust.ac.bw

J. B. H. Njagarah

njagarahh@biust.ac.bw

Y. A. Terefe

yibeltal.terefe@ul.ac.za

1 Department of Mathematics and Statistical Sciences, Botswana International University of Science and Technology (BIUST), Private Bag 016, Palapye, Botswana

2 Department of Mathematics and Applied Mathematics, University of Limpopo, Limpopo, South Africa 
curbing new infections, it ought to be done in conjunction with implementation of other control measures if the disease is to be curtailed in a shorter period of time. More still, when an effective vaccine is available, even a $98 \%$ coverage in one community may not be enough to completely stop new infections in the presence of migration. We therefore recommend that vaccination ought to be done universally if the daily number of new infections is to be reduced to zero.

Keywords COVID-19 · Self-protection · NPIs · Global stability · Sensitivity analysis · Mitigation strategy

\section{Introduction}

The Severe Acute Respiratory Syndrome-Coronavirus-2 (SARS-Cov-2) was first reported in Wuhan-Hubei Province, China on December 31, 2019 when it was reported to the World Health Organisation as respiratory disease causing acute Pneumonia [36]. The disease was code named COVID-19, (where COVID is the short form for coronavirus disease and the 19 indicating the year 2019 when it was first reported) [36]. COVID-19 is appearing as one of the most fatal human diseases reported in the world's history, it has caused a global health emergency, and has a clear potential for a long-lasting global pandemic with high fatality rates.

The disease affects people differently with some developing mild symptoms while others developing severe symptoms and needing hospitalisation. The most common symptoms in the early stages of the infection include fever, dry cough, flu like symptoms, fatigue, hyposmia/anosmia, loss of taste and appetite among others [21], with such symptoms appearing within 2-14 days after exposure. The detailed symptoms of the disease are given in [16,21]. The persons with co-morbidities, those with suppressed immunity, as well as the elderly are at risk of severe infection. The co-morbidities and the risk levels are detailed in [21].

The first COVID-19 case in South Africa was identified on the 5th of March 2020 as an imported case (see [26]), while the first locally transmitted case was detected on March 12, 2020 (see [22]). Since then the number of cases has continued to grow. As of August 16, 2020 (at 11:10 GMT) at 583, 653 cumulative case (approximately $52.4 \%$ of all reported cases in Africa), 466, 941 recoveries (56.4\% of recoveries on the continent), 11, 677 fatalities $(45.99 \%$ of reported COVID-19 related fatalities in Africa) [37]. In terms of the total number of reported cases, South Africa sits at the fifth high ranking countries behind Russia, India, Brazil and USA (with USA having reported the highest number of cases totalling to 5, 531, 065 which is approximately $25.56 \%$ of the total cases in the world as August 16, 2020 at the time of drafting this manuscript).

A number of articles studying the transmission dynamics of the disease in various communities/countries and regions have been appearing online since February 2020. One aspect that is clear at the moment (at the time of drafting this manuscript) is that the disease has no confirmed curing medicine for it and nor vaccine that can be used to prevent it. We note however that several countries around the world and laboratories have embarked on an audacious task of trying to find a vaccine so as to contain the global pandemic. In the absence of any pharmaceutical intervention, most governments have embarked on non-pharmaceutical interventions (NPIs) including: (i) wearing of face masks (covering the nose and mouth, with some people using face shields or both), (ii) frequent washing of hands with soap and clean water, or using alcohol based hand sanitizer (with at least $70 \%$ alcohol content), (iii) physi- 
$\mathrm{cal} /$ social distancing which involves maintaining a distance of at least $2 \mathrm{~m}$ between any two individuals as well as avoiding big gatherings most especially in closed spaces, (iv) continued disinfecting of public spaces since the virus can remain viable in the environment for several hours or even days.

When there does not exist a curing medicine or a working vaccination to prevent a contagious disease, the main possible targets in the fight against such a disease are to reduce (1) the number of adequate contacts of the population that lead to infection, (2) the transmission probability of the disease per each adequate contact, and (3) the average duration of infectiousness of infected individuals. For instance, in the case of COVID-19, the use of face masks, frequent hand washing and sanitizing, and disinfecting the environment contribute to the reduction of the transmission probability of the disease, whereas the lock down measures, social distancing ("physical distancing") and isolating/quarantining the exposed individuals help to reduce potentially risky contacts. Moreover, increasing the quantity and quality of testing to detect and isolate/quarantine infected individuals contribute to the reduction of the average duration of infectiousness of those who are infected and the possibility of passing the disease to others. Similar to other viral infections, "pre-symptomatic" and asymptomatic infected individuals pose a great risk as super spreaders. For the persons who later become symptomatic, detection and testing are usually done at the onset of the symptoms. In systems where testing is done upon onset of symptoms, the completely asymptomatic individuals will go undetected and these will pose a higher transmission risk compared to the symptomatic individuals.

Similar to other SARS-CoV infections and many respiratory infections, the novel SARS$\mathrm{CoV}-2$ is mainly transmitted through tiny respiratory droplets released from an infected individual through bodily secretions such as saliva and nasal fluid, contact with soiled hands, and the settling of aerosolized viral particles and droplets spread via talking, sneezing, coughing, and vomiting [27]. The main route of entry of the virus into the body is through the soft tissue lining the mouth, eyes and the nose. Once infected, individuals with COVID-19 can shed viral particles before, during, and after developing symptoms [19,30]. The respiratory droplets are believed not to travel distances of more than $2 \mathrm{~m}$ and are less likely to be suspended in the air for long periods of time. There may be exceptions however, given the wind strength, humidity and air conditioning most especially in closed spaces. The viral particles that are shed from an infected individual can be directly deposited onto the surrounding surfaces and some tiny particles can be re-suspended due to natural airflow patterns, mechanical airflow patterns, or other sources of turbulence in the environment $[11,27]$. When an individual makes contact with such contaminated surfaces, there is an exchange of microbial life, including a transfer of viruses from the individual to the surface and vice versa $[31,33]$.

Knowledge of the transmission dynamics of COVID-19 is currently developing, but based upon studies of SARS and MERS-CoV, preliminary data on SARS-CoV-2, and CDC recommendations, it is likely that SARS-CoV-2 can persist on fomites ranging from a couple of hours to 5 days $[4,12,34]$ depending on the material [34]. Based upon preliminary studies of SARS-CoV-2 survival, the virus survives longest at a relative humidity of $40 \%$ on plastic surfaces (half-life median $=15.9 \mathrm{~h}$ ) and shortest in aerosol form (half-life median $=2.74 \mathrm{~h}$ ) [34]. However, survival in aerosol was determined at a relative humidity of $65 \%$. Based on data related to SARS and MERS, we predict that the viability of SARS-CoV-2 in aerosol is likely longer at lower relative humidity levels. Survival of SARS-CoV-2 at $40 \%$ relative humidity on copper (half-life median $=3.4 \mathrm{~h}$ ), cardboard (half-life median $=8.45 \mathrm{~h}$ ), and steel (half-life median $=13.1 \mathrm{~h}$ ) collectively fall between survival in the air and on plastic [34]. However, it should be noted that there are no documented cases thus far of a COVID-19 infection originating from fomites. There is preliminary data demonstrating the presence of SARS-CoV-2 
in stool, indicating that transmission can potentially occur through the fecal-oral pathway $[27,28,39]$. While transmission of COVID-19 has been documented only through respiratory droplet spread and not through deposition on fomites, steps should still be taken to clean and disinfect all potential sources of SARS-CoV-2 under the assumption that active virus may be transmitted by contact with these abiotic surfaces [4,27]. With an abundance of caution, it is important to consider the possibility that the virus is transmitted through aerosols and surfaces [18].

Among the mathematical approaches used so far, many authors relied on deterministic compartmental models. This approach has been revealed successful for reproducing epidemic curves in the past SARS-CoV outbreak in 2002-2003 (see [9]) and has been employed also for COVID-19. The model construction addressing the dynamics of the disease caused by SARS-CoV-2 virus in specific localities should be informed by the protocol followed when managing the disease. For the case of South Africa, persons who test positive and show no or mild symptoms for COVID-19 are directed to self-isolate in their homes. However, those who show moderate to severe symptoms would usually require hospitalisation including admission into the Intensive Care Units (ICU).

Research papers describing mathematical models that study the disease dynamics in South Africa continue to be published. Here we cite a few. In the model projection in [23], an SEIR type model was used in the early projections of the disease. We note however that the model did not account for some special features such as the asymptomatic individuals, the contribution from contaminated surfaces/environment as well as quarantining/isolation of detected positive cases. In the paper by Mushayabasa et al. [24], the force of infection considered assumed that the level of contribution to new infections by undetected symptomatic individuals as well as persons with severe symptoms to be the same as that of asymptomatic individuals. This assumption does not address the issue of asymptomatic individuals being considered as super spreaders [25]. In addition, no environmental contribution was considered. On the other hand, the study by Garba et al. [8] accounted for the contribution of the environmental contamination to the disease transmission using mass-action form while the contribution from human-to-human transmission was modelled using standard-incidence formulation.

In this paper, the contribution of environmental contamination is modelled using a saturation term, which is consistent in terms of magnitude and unit of measurement to that of standard incidence formulation. Moreover, the behavioural change of the population is included in accordance with the "learning" associated with a class of susceptible individuals who practice self-protection measures thereby reducing the likelihood of contracting the infection. In addition, the model extensions highlight the dangers that may be associated with uncontrolled migration on the disease severity and the prospects of curtailing new infections. More still, scenarios of the possible outcomes of localised vaccination coverage (once the vaccine is available) in presence of migration are explored. The model is numerically solved for selected sets of nominal values of the parameters to ascertain the long term dynamics of the disease and possible implications of varying vital processes on the disease dynamics.

The rest of this paper is organised as follows. In Sect. 2, we describe the baseline model used, analyse the basic properties of the model including computation of the reproduction number, determination of the conditions under which equilibrium points exist. The model is slightly modified in Sect. 3 to include an additional class that accommodates all hospitalisations and isolated/quarantined individuals. The model is fitted to data and important parameter values are determined in Sect. 3.1. Numerical simulations of the extended model are presented in Sect. 4 followed by the discussion about the contribution of immigration and vaccination in Sect. 5. Finally, the paper is concluded in Sect. 6. 


\section{Baseline model and analysis}

We consider a baseline model of SEIR (Susceptible-Exposed-Infectious-Recovered) type with behaviour modification of the host population.

Here, it is assumed that the disease has no reinfections for the duration of the modeling time and transmission is from two sources: (1) direct (from human to human) and (2) indirect (from the contaminated environment). By combining the direct and indirect way of transmissions, the force of infection is assumed to have the form

$$
\lambda=\beta_{1} \frac{I+v A}{N}+\beta_{2} \frac{W}{K+W},
$$

where $N=S+S_{e}+E+A+I+R$ and $K$ is the concentration of the novel coronavirus in the environment which increases $50 \%$ chance of triggering the disease transmission. The description of the state variables and parameters involved in the model formulation are presented in Table 1. We assume the following system of nonlinear differential equations represents the transmission dynamics of COVID-19 in a particular population.

$$
\begin{aligned}
\dot{S} & =\pi-(\lambda+\sigma e+\mu) S, \\
\dot{S}_{e} & =\sigma e S-(1-\rho) \lambda S_{e}-\mu S_{e}, \\
\dot{E} & =\lambda S+(1-\rho) \lambda S_{e}-(\eta+\mu) E, \\
\dot{A} & =\ell \eta E-\left(\theta+\gamma_{1}+\mu\right) A, \\
\dot{I} & =(1-\ell) \eta E+\theta A-\left(\gamma_{2}+\delta+\mu\right) I, \\
\dot{R} & =\gamma_{1} A+\gamma_{2} I-\mu R, \\
\dot{W} & =\xi_{1} A+\xi_{2} I-\psi W,
\end{aligned}
$$

where $W$ represents the class of contaminated environmental and the dot sign is representing the time derivative of the indicated variable.

Following a similar approach as in [13-15], in this paper, we assume the behaviour of individuals in adopting self protective mechanisms changes upon receiving information about the daily confirmed new infections of the disease, and is given by the formula

$$
e(E)=\frac{(\eta E)^{n}}{E_{*}^{n}+(\eta E)^{n}}=\frac{E^{n}}{\left(\frac{1}{\eta} E_{*}\right)^{n}+E^{n}},
$$

where $E_{*}$ is the count of newly emerged infections corresponding to the threshold incidence in which individuals start reacting swiftly (that means, the point at which the behaviour change function changes its concavity). We appended the following nonnegative initial conditions with the system (2):

$$
\begin{aligned}
& S(0)=S_{0}, S_{e}(0)=S_{e_{0}}, E(0)=E_{0}, A(0)=A_{0}, \\
& I(0)=I_{0}, R(0)=R_{0}, \quad \text { and } W(0)=W_{0} .
\end{aligned}
$$

\subsection{Basic properties of the baseline model}

The model (2) has a disease free equilibrium given by

$$
\mathcal{E}_{0}=\left(\frac{\pi}{\mu}, 0,0,0,0,0,0\right) .
$$


Table 1 Description of the state variables and parameters in the model

\begin{tabular}{|c|c|}
\hline Variables & \multirow{2}{*}{$\begin{array}{l}\text { Description } \\
\text { Susceptible population }\end{array}$} \\
\hline $\mathrm{S}$ & \\
\hline$S_{e}$ & Susceptible individuals who are educated to prevent the disease \\
\hline $\mathrm{E}$ & Exposed individuals \\
\hline A & Asymptomatic infectious individuals \\
\hline I & Symptomatic infectious individuals \\
\hline $\mathrm{R}$ & Recovered individuals \\
\hline W & Corona virus contaminated surfaces or objects in the environment \\
\hline Parameters & Description \\
\hline$\pi$ & Rate of local recruitment to the susceptible individuals \\
\hline$\mu$ & Natural death rate \\
\hline$\beta_{1}$ & Contact rate among human population \\
\hline$\beta_{2}$ & Contact rate with $W$ class \\
\hline$v_{1}, v_{2}$ & Modification parameters \\
\hline$K$ & The concentration of the disease pathogen in the environment \\
\hline$\sigma$ & Rate of dissemination of information about the disease in the population \\
\hline$\rho$ & Average effectiveness of existing self-preventive measures \\
\hline$\eta$ & Progression to infectiousness \\
\hline$\ell$ & Fraction of infected individuals who become symptomatic \\
\hline$\theta$ & Rate of asymptomatic individuals develop symptoms and transfer to the $I$ class \\
\hline$\gamma_{1}, \gamma_{2}$ & Rate of recovery \\
\hline$\delta$ & Death rate due to corona virus \\
\hline$\psi$ & Rate of disinfecting touched objects and surfaces in the environment \\
\hline$\xi_{1}, \xi_{2}$ & Shedding rate of infected class to the environment \\
\hline
\end{tabular}

Theorem 1 The model system (2) is biological significance on the region given by

$$
\Omega=\Omega_{H} \times \Omega_{W}
$$

where $\Omega_{H} \in \mathbb{R}^{6}$ and $\Omega_{W} \in \mathbb{R}$ such that

$$
\Omega_{H}=\left\{\left(S, S_{e}, E, A, I, R\right) \in \mathcal{R}^{6}: 0 \leq S+S_{e}+E+A+I+R=N<\frac{\pi}{\mu}\right\}
$$

and

$$
\Omega_{W}=\left\{W \in \mathbb{R} \mid W<\frac{\left(\xi_{1}+\xi_{2}\right) \pi}{\mu \psi}\right\} .
$$

Proof Since the model analyses the change in populations, it is necessary to show that all solutions of Eq. (2) are non-negative as required in [2]. To show that the state variables $S$ and $S_{e}$ of the model are positive for all $t \geq 0$, we use proof by contradiction. We suppose that a trajectory crosses one of the positive cones at times $t_{1}$ or $t_{2}$ such that:

$$
\begin{aligned}
& -t_{1}: S\left(t_{1}\right)=0, \dot{S}\left(t_{1}\right)<0, S_{e}(t)>0, E(t)>0, A(t)>0, I(t)>0, R(t)>0, \text { and } \\
& \quad W(t)>0 \text { for } t \in\left(0, t_{1}\right), \text { or }
\end{aligned}
$$


$-t_{2}: S_{e}\left(t_{2}\right)=0, \dot{S}_{e}\left(t_{2}\right)<0, S(t)>0, E(t)>0, A(t)>0, I(t)>0, R(t)>0$, and $W(t)>0$ for $t \in\left(0, t_{2}\right)$,

Using the first equation of (2), the first assumption leads to

$$
\dot{S}\left(t_{1}\right)=0 \geq 0
$$

which contradicts the assumption that $\dot{S}\left(t_{1}\right)<0$. Therefore, $S(t)$ remains non-negative for all $t>0$.

Considering the second assumption and second equation of (2), we have

$$
\dot{S}\left(t_{2}\right)=\sigma e S>0 . \sigma, e>0
$$

which contradicts the assumption that $\dot{S}\left(t_{2}\right)<0$. Hence, $S_{e}(t)$ remains non-negative for all $t>0$.

Based on the third equation of (2), we have

$$
\dot{E}(t)=\lambda S+(1-\rho) \lambda S_{e}-(\eta+\mu) E \geq-(\eta+\mu) E .
$$

The resulting equation (8) yields

$$
E(t) \geq E(0) e^{-(\eta+\mu) t} \geq 0 .
$$

Similarly, for the last fourth equations of (2), we have

$$
\left\{\begin{array}{l}
A(t) \quad \geq A(0) e^{-\left(\theta+\gamma_{1}+\mu\right) t} \geq 0, \\
I(t) \geq I(0) e^{-\left(\mu+\gamma_{2}+\delta\right) t} \geq 0, \\
R(t) \geq R(0) e^{-\mu t} \geq 0, \\
W(t) \geq W(0) e^{-\psi t} \geq 0 .
\end{array}\right.
$$

Therefore, the state variables of the model system (2) are non-negative for all $t>0$.

The equation for the change in the total population is obtained by adding the first six equations of (2) such that

$$
\dot{N}(t)=\pi-\mu N-\delta I \leq \pi-\mu N .
$$

The solution to the resulting inequality is given by

$$
N(t) \leq \frac{\pi}{\mu}+\left(N(0)-\frac{\pi}{\mu}\right)^{-\mu t} .
$$

Hence, considering the initial population such that $0 \leq N(0) \leq \frac{\pi}{\mu}$, by Gronwall inequality, we have

$$
0 \leq N(t) \leq \frac{\pi}{\mu}
$$

Considering the free virus in the environment, we have

$$
\dot{W}(t)=\xi_{1} A+\xi_{2} I-\psi W \leq\left(\xi_{1}+\xi_{2}\right) \frac{\pi}{\mu}-\psi W,
$$

which on applying the Gronwall inequality, for the initial conditions $0 \leq W(0) \leq \frac{\left(\xi_{1}+\xi_{2}\right) \pi}{\mu \psi}$, leads to

$$
0 \leq W(t) \leq \frac{\left(\xi_{1}+\xi_{2}\right) \pi}{\mu \psi}
$$


Therefore, both the human population under consideration and the density of the pathogen are non-negative and bounded. Combining the above two steps in line with Theorem 2.1.5 in [32] describing the existence of unique bounded solutions, then the domain of biological significance $\Omega$ is positively invariant.

\subsubsection{Baseline model basic reproduction number}

To find the model basic reproduction number denoted by $\mathcal{R}_{0}$, we use the next generation matrix method which is detailed in [35] and found out

$$
\mathcal{R}_{0}=\frac{\beta_{1}\left(\ell \eta \theta+(1-\ell) \eta k_{2}\right)}{k_{1} k_{2} k_{3}}+\frac{\beta_{1} v \ell \eta}{k_{1} k_{2}}+\frac{\beta_{2} \pi\left[\ell \eta \xi_{1} k_{1}+\ell \eta \theta \xi_{2}+(1-\ell) \eta k_{2} \xi_{2}\right]}{k_{1} k_{2} k_{3} \mu \psi K},
$$

where $k_{1}=\eta+\mu, \quad k_{2}=\theta+\gamma_{1}+\mu$, and $k_{3}=\gamma_{2}+\mu+\delta$.

The next result follows from Theorem 2 of [2].

Theorem 2 The DFE $\mathcal{E}_{0}$ of the model (2) is locally asymptotically stable whenever $\mathcal{R}_{0}<1$ and unstable if $\mathcal{R}_{0}>1$.

The epidemiological implication of Theorem 2 is that the transmission of coronavirus can be controlled by having $\mathcal{R}_{0}<1$ if the initial total numbers of the sub-populations involved in Eq.(2) are in the basin of attraction of $\mathcal{E}_{0}$. To ensure that eliminating the disease is independent of the initial size of the subpopulation, the disease-free equilibrium must be globally asymptotically stable when $\mathcal{R}_{0}<1$. This is what we present next.

Theorem 3 The disease free equilibrium $\mathcal{E}_{0}$ is globally asymptotically stable whenever $\mathcal{R}_{0}$ is less than unity.

Proof To prove global stability of the disease free equilibrium $\mathcal{E}_{0}$ we use a suitably defined Lyapunov function. We consider a $\mathcal{C}^{1}$ candidate Lyapunov function $G$ such that

$$
\mathcal{G}=a E+b A+c I+d W
$$

where $a, b, c$ and $d$ are non-negative constants to be determined. The derivative of the Lyapunov function is given by

$$
\begin{aligned}
\dot{\mathcal{G}}= & a \dot{E}+b \dot{A}+c \dot{I}+d \dot{W}, \\
= & a\left[\lambda S+(1-\rho) \lambda S_{e}-k_{1} E\right]+b\left[\ell \eta E-k_{2} A\right]+c\left[(1-\ell) \eta E+\theta A-k_{3} I\right] \\
& +d\left[\xi_{1} A+\xi_{2} I-\psi W\right], \\
\leq & a\left[\beta_{1}(I+v A)+\frac{\beta_{2} W \frac{\pi}{\mu}}{K}-k_{1} E\right] \\
& +b\left[\ell \eta E-k_{2} A\right]+\left[(1-\ell) \eta E+\theta A-k_{3} I\right]+d\left[\xi_{1} A+\xi_{2} I-\psi W\right], \\
= & {\left[-k_{1} a+b \eta \ell+(1-\ell) \eta c\right] E+\left[a \beta_{1} v-b k_{2}+c \theta+d \xi_{1}\right] A+\left[a \beta_{1}-c k_{2}+d \xi_{2}\right] I } \\
& +\left[a \frac{\beta_{2} \frac{\pi}{\mu}}{K}-d \psi\right] W .
\end{aligned}
$$


We equate the coefficients of $E, I$ and $W$ to zero and letting $c=1$, we obtain the values,

$$
\left\{\begin{array}{l}
c=1 \\
b=\frac{\left(\beta_{1} \nu(1-\ell) \eta \psi K+\theta k_{1} \psi K+\beta_{2}(1-\ell) \eta \xi_{1} \frac{\pi}{\mu}\right)}{k_{1} k_{1} \psi K-\left(\beta_{1} \nu \ell \eta \psi K+\beta_{2} \ell \eta \xi_{1} \frac{\pi}{\mu}\right)} \\
a=\frac{b \ell \eta+(1-\ell) \eta}{k_{1}} \\
d=\frac{\beta_{2}(b \ell \eta+(1-\ell) \eta) \frac{\pi}{\mu}}{k_{1} \psi K}
\end{array}\right.
$$

Using the coefficients obtained in (17), the derivative of the Lyapunov function reduces to

$$
\dot{\mathcal{G}} \leq-\psi^{2} k_{1}^{2} K^{2} k_{2} k_{3}\left(1-\mathcal{R}_{0}\right) I .
$$

We note that when $\mathcal{R}_{0}<1, \dot{\mathcal{G}}<0$ and equality is achieved when $\mathcal{R}_{0}=1$ or $I=0$ which is at the disease free equilibrium. By the LaSalle invariance principle (see [17]), the equilibrium $\mathcal{E}_{0}$ is globally asymptotically stable.

\subsection{Endemic equilibrium of the baseline model}

To determine the endemic equilibrium of the baseline model at $E_{1}=\left(S^{*}, S_{e}^{*}, E^{*}, A^{*}, I^{*}\right.$, $\left.R^{*}, W^{*}\right)$, we equate the left hand side of the model system (2) to zero and obtain

$$
\begin{aligned}
& 0=\pi-\left(\lambda^{*}+\sigma e^{*}\right) S^{*}-\mu S^{*}, \\
& 0=\sigma e^{*} S^{*}-(1-\rho) \lambda^{*} S_{e}^{*}-\mu S_{e}^{*}, \\
& 0=\lambda^{*} S^{*}+(1-\rho) \lambda^{*} S_{e}^{*}-k_{1} E^{*}, \\
& 0=\ell \eta E^{*}-k_{2} A^{*}, \\
& 0=(1-\ell) \eta E^{*}+\theta A^{*}-k_{3} I^{*}, \\
& 0=\gamma_{1} A^{*}+\gamma_{2} I^{*}-\mu R^{*}, \\
& 0=\xi_{1} A^{*}+\xi_{2} I^{*}-\psi W^{*} .
\end{aligned}
$$

From equations (18d), (18e), (18f) and (18g),

$$
\begin{aligned}
A^{*} & =\Phi_{1} E^{*}, \text { where } \Phi_{1}=\frac{\ell \eta}{k_{2}}, \\
I^{*} & =\Phi_{2}^{*} E^{*}, \text { where } \Phi_{2}=\frac{(1-\ell) \eta+\theta \Phi_{1}}{k_{3}}, \\
R^{*} & =\Phi_{3} E^{*}, \text { where } \Phi_{3}=\frac{\gamma_{1} \Phi_{1}+\gamma_{2} \Phi_{2}}{\mu}, \\
W^{*} & =\Phi_{4} E^{*}, \text { where } \Phi_{4}=\frac{\xi_{1} \Phi_{1}+\xi_{2} \Phi_{2}}{\psi} .
\end{aligned}
$$

Adding up the equations (18a), (18b) and (18c), and using the expression for the total population $N$, we obtain

$$
N^{*}=\frac{\pi}{\mu}-\Phi_{5} E^{*}, \text { where } \Phi_{5}=\frac{\eta}{\mu}-\left(\Phi_{1}+\Phi_{2}+\Phi_{3}\right) .
$$

Using the expression for the force of infection at equilibrium $\lambda^{*}$ and substituting the appropriate terms, we obtain 


$$
\lambda^{*}=\frac{\left(\Phi_{6}-\Phi_{7} E^{*}\right) E^{*}}{\frac{\pi}{\mu} K+\Phi_{8} E^{*}-\Phi_{9}\left(E^{*}\right)^{2}},
$$

where

$$
\left\{\begin{array}{l}
\Phi_{6}=\beta_{1}\left(\Phi_{2}+v \Phi_{1}\right) K+\beta_{2} \Phi_{4} \frac{\pi}{\mu} \\
\Phi_{7}=\left[\beta_{2} \Phi_{5}-\beta_{1}\left(\Phi_{2}+v \Phi_{1}\right)\right] \Phi_{4} \\
\Phi_{8}=\frac{\pi}{\mu} \Phi_{4}-\Phi_{5} K \\
\Phi_{9}=\Phi_{4} \Phi_{5} .
\end{array}\right.
$$

From equation (18c), we can express $S_{e}^{*}$ in terms of $S^{*}$ as $E^{*}$ such that

$$
S_{e}^{*}=\frac{k_{1} E^{*}-\lambda^{*} S^{*}}{(1-\rho) \lambda^{*}} .
$$

Substituting for $S_{e}^{*}$ in (18b), we obtain

$$
S^{*}=\frac{\left[(1-\rho) \lambda^{*} k_{1}+\mu k_{1}\right] E^{*}}{\left[(1-\rho)\left(\sigma e^{*}+\lambda^{*}\right)+\mu\right] \lambda^{*}} .
$$

Substituting for $S^{*}$ in equation (18a), we obtain

$$
\pi\left[(1-\rho)\left(\sigma e^{*}+\lambda^{*}\right)+\mu\right] \lambda^{*}=\left(\lambda^{*}+\sigma e^{*}+\mu\right)\left[(1-\rho) \lambda^{*} k_{1}+\mu k_{1}\right] E^{*} .
$$

We note from the expression (24) that $\lambda^{*}$ is expressed in terms of $E^{*}$. On substituting for $\lambda^{*}$ and $e^{*}$ into equation (26), we obtain one of the roots as $E^{*}=0$, which corresponds to the disease free equilibrium. When $E^{*} \neq 0$, noting that $E_{*}$ is constant, we obtain a polynomial of the order $n+4$ given by

$$
\begin{aligned}
& E^{* n}\left[C_{4} E^{* 4}+C_{3} E^{* 3}+C_{2} E^{* 2}+C_{1} E^{*}+C_{0}\right] \\
& +\left(\frac{1}{\eta} E_{*}\right)^{n}\left[D_{4} E^{* 4}+D_{3} E^{* 3}+D_{2} E^{* 2}+D_{1} E^{*}+D_{0}\right]=0,
\end{aligned}
$$

where the coefficients $C_{i}$ for $i=1,2,3,4$ are such that

$$
\begin{aligned}
C_{4}= & k_{1} \mu(\mu+\sigma) \Phi_{9}^{2}+(1-\rho) k_{1} \Phi_{7}^{2}+k_{1}[(1-\rho)(\sigma+\mu)+\mu] \Phi_{7} \Phi_{5} \Phi_{4} \\
C_{3}= & -2(1-\rho) k_{1} \Phi_{6} \Phi_{7}-k_{1}((1-\rho)(\sigma+\mu)+\mu)\left(\Phi_{7} \Phi_{8}+\Phi_{6} \Phi_{9}\right)-(1-\rho) \pi \Phi_{7}^{2} \\
& -\pi((1-\rho) \sigma+\mu) \Phi_{7} \Phi_{9}-2 \mu k_{1} \Phi_{8} \Phi_{9}(\sigma+\mu), \\
C_{2}= & (1-\rho) k_{1} \Phi_{6}+2(1-\rho) \pi \Phi_{6} \Phi_{7}+k_{1}((1-\rho)(\mu+\sigma)+\mu)\left(\Phi_{6} \Phi_{8}-\frac{\pi}{\mu} K \Phi_{7}\right) \\
& +\pi(\mu+(1-\rho) \sigma)\left(\Phi_{7} \Phi_{8}+\Phi_{6} \Phi_{9}\right)+k_{1} \mu(\mu+\sigma)\left(\Phi_{8}^{2}-2 \frac{\pi}{\mu} K \Phi_{9}\right), \\
C_{1}= & \frac{\pi}{\mu} k_{1} \Phi_{6} K((1-\rho)(\sigma+\mu)+\mu)-(1-\rho) \pi \Phi_{6}-\pi(\mu+(1-\rho) \sigma)\left(\Phi_{6} \Phi_{8}-\frac{\pi}{\mu} K \Phi_{7}\right) \\
& 2 \pi k_{1} K(\sigma+\mu) \Phi_{8}, \\
C_{0}= & k_{1} \pi^{2} K^{2}+\sigma k_{1} \frac{\pi^{2}}{\mu} K^{2}-\mu \pi \Phi_{6} \frac{\pi}{\mu} K-(1-\rho) \pi \sigma \Phi_{6} \frac{\pi}{\mu} K,
\end{aligned}
$$

and the coefficients $D_{i}$ for $i=1,2,3,4$ are given by

$$
\begin{aligned}
D_{4}= & (1-\rho) k_{1} \Phi_{7}^{2}+\mu(2-\rho) k_{1} \Phi_{7} \Phi_{9}+\mu^{2} k_{1} \Phi_{9}^{2} \\
D_{3}= & -2(1-\rho) k_{1} \Phi_{6} \Phi_{7}-(1-\rho) \pi \Phi_{7}^{2}-\mu(2-\rho) k_{1}\left(\Phi_{7} \Phi_{8}+\Phi_{6} \Phi_{9}\right) \\
& -\mu \pi \Phi_{7} \Phi_{9}-2 \mu^{2} k_{1} \Phi_{8} \Phi_{9},
\end{aligned}
$$




$$
\begin{aligned}
D_{2}= & (1-\rho) k_{1} \Phi_{6}+2(1-\rho) \pi \Phi_{6} \Phi_{7}+\mu(2-\rho) k_{1}\left(\Phi_{6} \Phi_{8}-\frac{\pi}{\mu} K \Phi_{7}\right) \\
& +\mu \pi\left(\Phi_{7} \Phi_{8}+\Phi_{6} \Phi_{9}\right)+\mu^{2} k_{1}\left(\Phi_{8}^{2}-2 \frac{\pi}{\mu} K \Phi_{9}\right), \\
D_{1}= & (2-\rho) \pi k_{1} \Phi_{6} K-(1-\rho) \pi \Phi_{6}+\mu \pi\left(\frac{\pi}{\mu} K \Phi_{7}-\Phi_{6} \Phi_{8}\right)+2 \mu \pi k_{1} K \Phi_{8} . \\
D_{0}= & \pi^{2} k_{1} K^{2}-\pi^{2} K \Phi^{6}=\pi^{2} k_{1} K^{2}\left(1-\mathcal{R}_{0}\right) .
\end{aligned}
$$

We note that $D_{0}$ is negative for $\mathcal{R}_{0}>1$ and positive when $\mathcal{R}_{0}<1$ whereas $C_{4}$ is always positive (not difficult to observe it after simplification). In addition, the total number of possible positive real roots of the polynomial (27) depends on the signs of the coefficients. To determine the total number of positive roots of equation (27), we use Descartes' rule of signs [7]. According to Descartes' rule of signs, total number of positive real zeros is either the same as the total number of sign changes of the coefficients of the polynomial or less than the total number of sign changes by even number. Hence, we state the next theorem for the existence of endemic equilibrium.

Theorem 4 The model system (2) has at least one endemic equilibrium when $\mathcal{R}_{0}>1$.

Theorem 5 For the model system (2), the endemic equilibrium guaranteed by Theorem 4 is locally asymptotically stable for $\mathcal{R}_{0}$ close to one.

The proof of Theorem 5 follows from the Center Manifold Theorem in [3].

\section{Model with isolation}

The baseline model described above does not consider the effect of isolating/quarantining and hospitalizing infected individuals as part of the dynamical process. However, practically individuals who tested positive for COVID-19 are being isolated/quarantined (and/or hospitalized) so that they do not further spread the disease to others. In this section we modify the baseline model to include the cohort of individuals, denoted by $Q$, who are isolated/quarantined after tested positive to the disease. Note that, hospitalized individuals have the same effect as the isolated/quarantined ones in terms of their ability to mix with others and thereby passing the infection. Therefore, we assume that hospitalized individuals are subsets of the cohort $Q$. The corresponding mathematical model is given in Eq. (28) below.

$$
\begin{aligned}
\dot{S} & =\pi-(\lambda+\sigma e+\mu) S, \\
\dot{S}_{e} & =\sigma e S-[(1-\rho) \lambda+\mu] S_{e}, \\
\dot{E} & =\lambda S+(1-\rho) \lambda S_{e}-(\eta+\mu) E, \\
\dot{A} & =\ell \eta E-\left(\theta+\phi_{1}+\gamma_{1}+\mu\right) A, \\
\dot{I} & =(1-\ell) \eta E+\theta A-\left(\phi_{2}+\gamma_{2}+\mu+\delta\right) I, \\
\dot{Q} & =\phi_{1} A+\phi_{2} I-\left(\gamma_{3}+\mu+\delta\right) Q, \\
\dot{R} & =\gamma_{1} A+\gamma_{2} I+\gamma_{3} Q-\mu R, \\
\dot{W} & =\xi_{1} A+\xi_{2} I-\psi W,
\end{aligned}
$$

In this model, the parameters $\phi_{1}$ and $\phi_{2}$ represent the rate at which people are tested to be positive from the asymptomatic and symptomatic cohorts and being transferred to the 
isolated/quarantined section, whereas $\gamma_{3}$ is the rate at which the isolated/quarantined individuals recover from the disease. Since the modified model has shown the same mathematical analysis as the baseline model and the value of $\mathcal{R}_{o}$ does not change, we omitted the presentation of the analysis here. However, we present the numerical simulations of this model to demonstrate the effect of some intervention mechanisms, especially the Non-Pharmaceutical interventions (NPIs) on the long term dynamics of the disease. To this effect, we first estimate the parameters involved in system equation (28) and analyse the sensitivity of the threshold values corresponding to any variation in the values of these parameters.

\subsection{Parameter estimation and sensitivity}

To estimate the values of parameters and study their sensitivity, the mathematical model system (28) is fitted to the new cases and disease related deaths data from South Africa.

\subsubsection{Parameter estimation}

The life expectancy of South Africa has been fluctuating with a major decline observed from 1990 to 2005 where it declined from 63.25 to 53.9. Since 2005, the life expectancy has been increasing and stands at 64.12 years in 2020 [20]. The estimated total population of South Africa is 59, 414, 253 [37]. We therefore use the value of $\pi$ such that $\pi=\mu \times N_{0}$. For detailed selection of ranges of parameters related to the biology of the disease, see [16]. The mean incubation period for the novel coronavirus has been reported at an average value of 5.2 days [4]. In some cases the incubation period has been observed to vary in a range of 2-14 days with outliers observed in a range of 0-27 days [38]. We select a sampling range of $(0.071,0.2)$ which is equivalent to the mean period of 5-14 days with our model fit giving the incubation period of about 6.2 days. The recovery rate of the infected individuals varies considerably depending on their immune fitness (influenced by co-morbidities and age, as mentioned in [4]) as well as severity of the disease. According to the CDC report in [5] patients who showed mild to moderate symptoms remained infectious for a period not longer than 10 days from the time symptoms were first detected, whereas those with more severe symptoms and those whose immune system was compromised remained infectious for no longer than 20 days. It should be noted that these are average values taken from various studies.

In this subsection, we shall consider the numerical simulation of the model against known data and then we will indicate the long term prediction of the dynamics of the disease in the population.

As can be seen from Fig. 1, the solution of the model fits very well with both the daily new cases data and the data for daily deaths. With the same continued state of the disease without much change, the prediction of the peak of the disease is after the first week of November 2020 as it can be observed from Fig. 2. This period is equivalent to about 250 days from when the first case was reported.

We note also that the asymptomatic new cases may not in most cases be detected but still largely wreak havoc as silent super-spreaders. The model simulations depict that all the subclasses would reach the peak around the same time. 

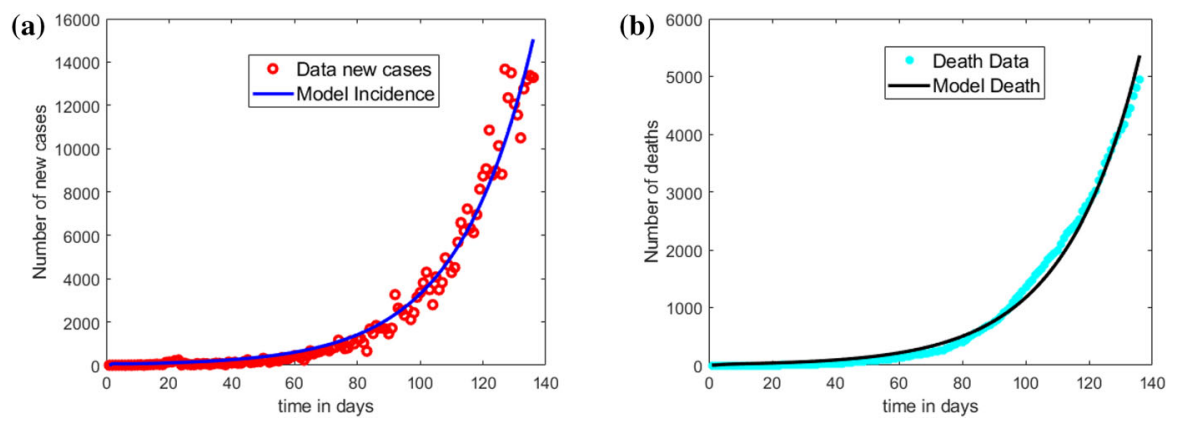

Fig. 1 Model fit to the data. a Model fitting with new cases data, $\mathbf{b}$ model fitting with daily death data

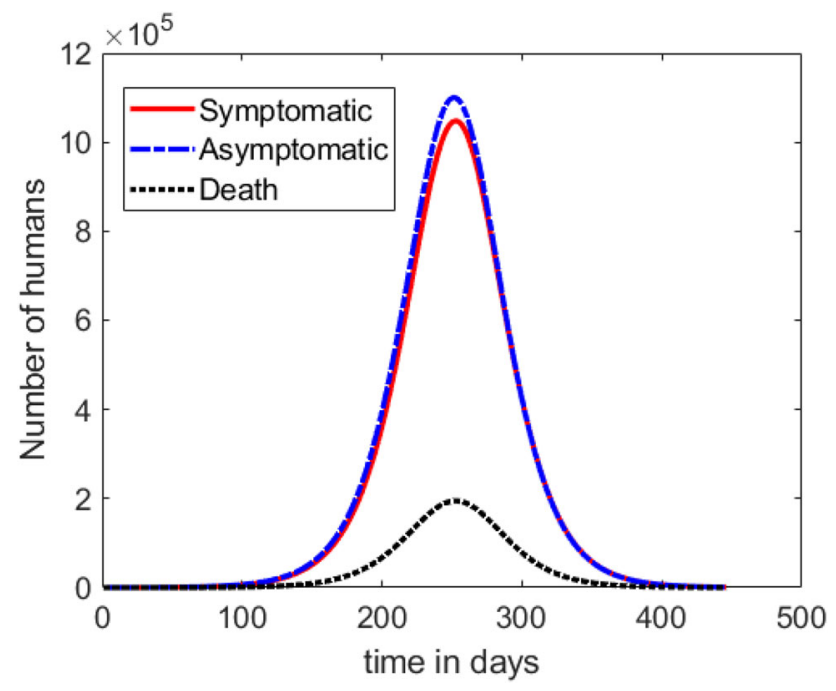

Fig. 2 Model simulation of the transmission depicting daily new cases

\subsubsection{Sensitivity analysis}

To carry out sensitivity analysis of the model input parameters to the model output, which in this case is the disease threshold. We use the Latin Hypercube sampling (LHS) scheme explained in $[1,10]$ to simultaneously sample the input parameter space and compute the values of the reproduction number as the output. The graphical representation of the PRCCs is indicated in the Tornado plot, Fig. 3a. For the sampled parameter space with the peak values as the nominal values indicated in Table 2, we produce a box plot (Fig. 3b) summarising key information about the computed reproduction number $\left(\mathcal{R}_{0}\right)$, that is, the minimum value, lower quartile, the median, the upper quartile, the observed maximum, and outliers.

The obtained values of $\mathcal{R}_{0}$ from the LHS scheme are in the range $(0.5,3)$. From the results depicted in the box plot, Fig. 3b, it is evident that although the median value of the computed reproduction number is greater than 1 , we can still find combinations of parameter values for which the reproduction number is less than 1 , in which case the disease can be contained. In the same respect, we can find combinations of parameter values for which the underlying 

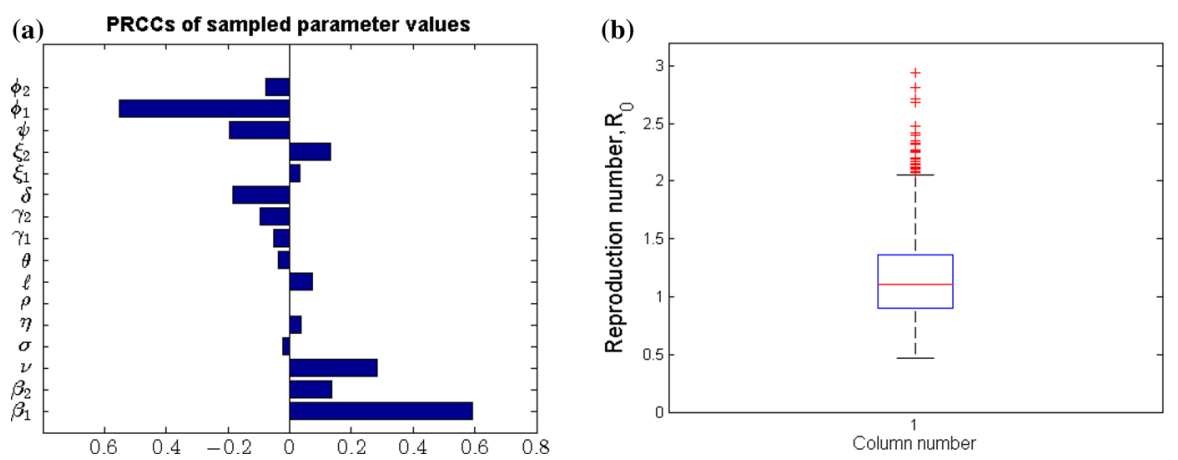

Fig. 3 PRCCs of sampled parameter values. a the Tornado plot summarising the PRCCs of the sampled parameter. The parameters with positive PRCCs indicate processes that can make the epidemic worse when increased and those with negative PRCCs can help contain the epidemic when increased. b gives the five-number (i.e., the minimum, lower quartile, median, upper quartile, and the maximum) summary of the computed values of $\mathcal{R}_{0}$ from the sampled parameter space

Table 2 Estimated baseline parameter values

\begin{tabular}{|c|c|c|c|c|}
\hline Parameter & Unit description & Range & Nominal value & Source \\
\hline$\pi$ & Persons day ${ }^{-1}$ & & $\mu \times N_{0}$ & {$[20,37]$} \\
\hline$\beta_{1}$ & contacts day $^{-1}$ & $(0.1274,0.2624)$ & 0.26097 & Fitted \\
\hline$\beta_{2}$ & contacts day ${ }^{-1}$ & $(0.00274,0.0100274)$ & 0.002787 & Fitted \\
\hline$v$ & Relative value & $(1.1,2.1)$ & 1.975 & Fitted \\
\hline$K$ & Pathogen concentration & $\left(10^{4}, 10^{7}\right)$ & $9,999,885$ & Fitted \\
\hline$\mu$ & day $^{-1}$ & & $(1 /(64.12 \times 365))$ & {$[20]$} \\
\hline$\sigma$ & day $^{-1}$ & $(0.18,0.67)$ & 0.637 & Fitted \\
\hline$\eta$ & day $^{-1}$ & $(0.071,0.20)$ & 0.1632 & Fitted \\
\hline$\rho$ & Proportion & $(0.32,0.475)$ & 0.3233 & Fitted \\
\hline$\ell$ & Proportion & $(0.48,0.83)$ & 0.8172 & Fitted \\
\hline$\theta$ & day $^{-1}$ & $(0.0714,0.1429)$ & 0.1141 & Fitted \\
\hline$\gamma_{1}$ & day $^{-1}$ & $(0.07,0.12)$ & 0.08385 & Fitted \\
\hline$\gamma_{2}$ & day $^{-1}$ & $(0.01,0.08)$ & 0.0761 & Fitted \\
\hline$\delta$ & day $^{-1}$ & $(0.10,0.235)$ & 0.1746 & Fitted \\
\hline$\xi_{1}$ & day $^{-1}$ & $(0.01,0.22)$ & 0.0148 & Fitted \\
\hline$\xi_{2}$ & day $^{-1}$ & $(0.01,0.35)$ & 0.02199 & Fitted \\
\hline$\psi$ & day $^{-1}$ & $(0.3333,3.4285)$ & 1.9119 & Fitted \\
\hline$\gamma_{3}$ & day $^{-1}$ & $(0.07,0.15)$ & 0.07343 & Fitted \\
\hline$\phi_{1}$ & day $^{-1}$ & $(0.01,0.30)$ & 0.0132 & Fitted \\
\hline$\phi_{2}$ & day $^{-1}$ & $(0.35,0.70)$ & 0.4194 & Fitted \\
\hline
\end{tabular}


Table 3 Parameter PRCC significance (FDR adjusted $p$-values)

\begin{tabular}{llll}
\hline Variable & PRCC & $p$ value & Keep \\
\hline$\beta_{1}$ & 0.5928 & 0.00000 & TRUE \\
$\beta_{2}$ & 0.1363 & $4.597 \times 10^{-05}$ & TRUE \\
$\nu$ & 0.2825 & 0.00000 & TRUE \\
$\sigma$ & -0.0205 & $5.553 \times 10^{-1}$ & FALSE \\
$\eta$ & 0.0353 & $3.130 \times 10^{-1}$ & FALSE \\
$\rho$ & -0.0005 & $9.875 \times 10^{-1}$ & FALSE \\
$\ell$ & 0.0732 & $3.450 \times 10^{-2}$ & TRUE \\
$\theta$ & -0.0385 & $3.032 \times 10^{-1}$ & FALSE \\
$\gamma_{1}$ & -0.0498 & $1.721 \times 10^{-1}$ & FALSE \\
$\gamma_{2}$ & -0.0972 & $4.492 \times 10^{-3}$ & TRUE \\
$\delta$ & -0.1857 & $1.254 \times 10^{-8}$ & TRUE \\
$\xi_{1}$ & 0.0349 & $3.130 \times 10^{-1}$ & FALSE \\
$\xi_{2}$ & 0.1327 & $6.574 \times 10^{-5}$ & TRUE \\
$\psi$ & -0.1932 & $3.486 \times 10^{-9}$ & TRUE \\
$\phi_{1}$ & -0.5522 & 0.00000 & TRUE \\
$\phi_{2}$ & -0.0788 & $2.372 \times 10^{-2}$ & TRUE \\
\hline
\end{tabular}

processes can make the epidemic worse. The $p$-values for the PRCCs are computed after performing Fisher transformation as explained in $[1,16]$. The results are indicated in Table 3.

For the parameters whose PRCCs with $p$-values less that 0.05 , we carry out pairwise comparison with the null hypothesis, $H_{0}$ being that the compared sensitive parameters are significantly different. The $p$-values are computed between the compared parameters. The results are summarised in Table 4.

To reduce the likelihood of making a Type 1 statistical error, False discovery rate adjusted $p$-values are computed and the results are summarised in Table 5. A more detailed explanation of the FDR process for a similar computation is given in [16].

The pairwise compared parameter values whose FDR adjusted $p$-values are less that 0.05 are observed to be significantly different and therefore the null hypothesis that "the compared parameters are significantly different" can not be rejected. The summary given in Table 6 is based on the results presented in Table 5 where "TRUE" indicates that the compared parameters are observed to be significantly different whereas the "FALSE" indicates that the compared parameters have $p$-value greater than 0.05 and therefore, the null hypothesis is rejected.

\subsection{Herd immunity}

Assuming that individuals recover from the disease with permanent immunity, the disease may eventually be blocked from being spread in the population if a certain proportion of the society is being recovered from the disease even though some few people are still susceptible. This phenomena is called the herd immunity of population. Herd immunity is a form of indirect protection for susceptible individuals in a population where having enough recovered (or vaccinated, if vaccination is available) individuals in the population decrease the number of susceptible hosts for an infectious agent, thus preventing or limiting the spread of disease 


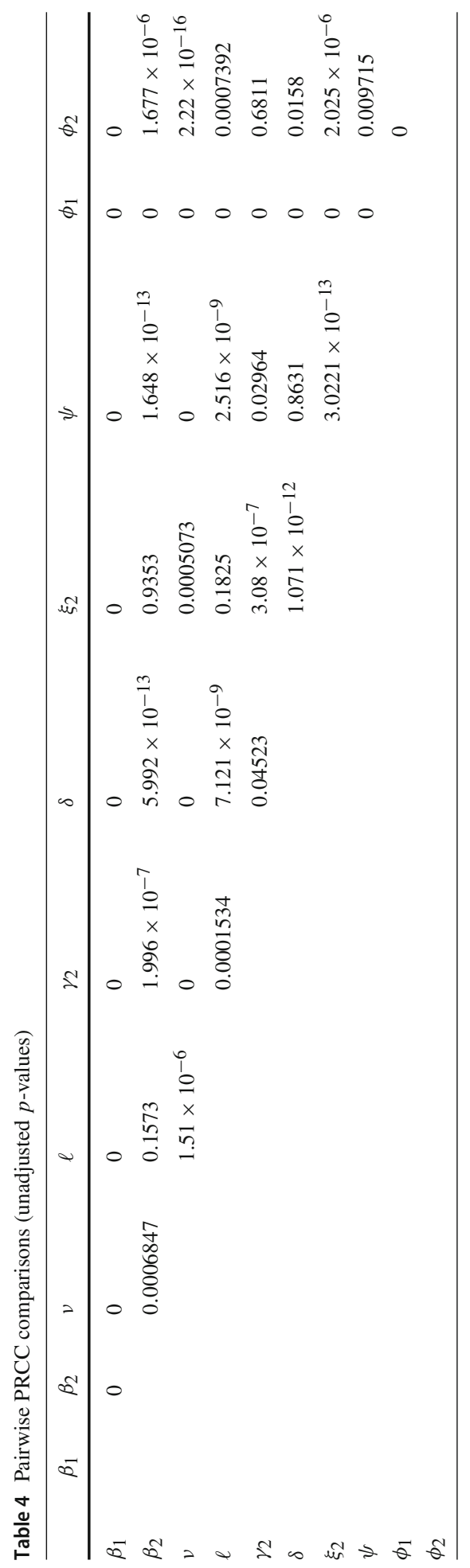




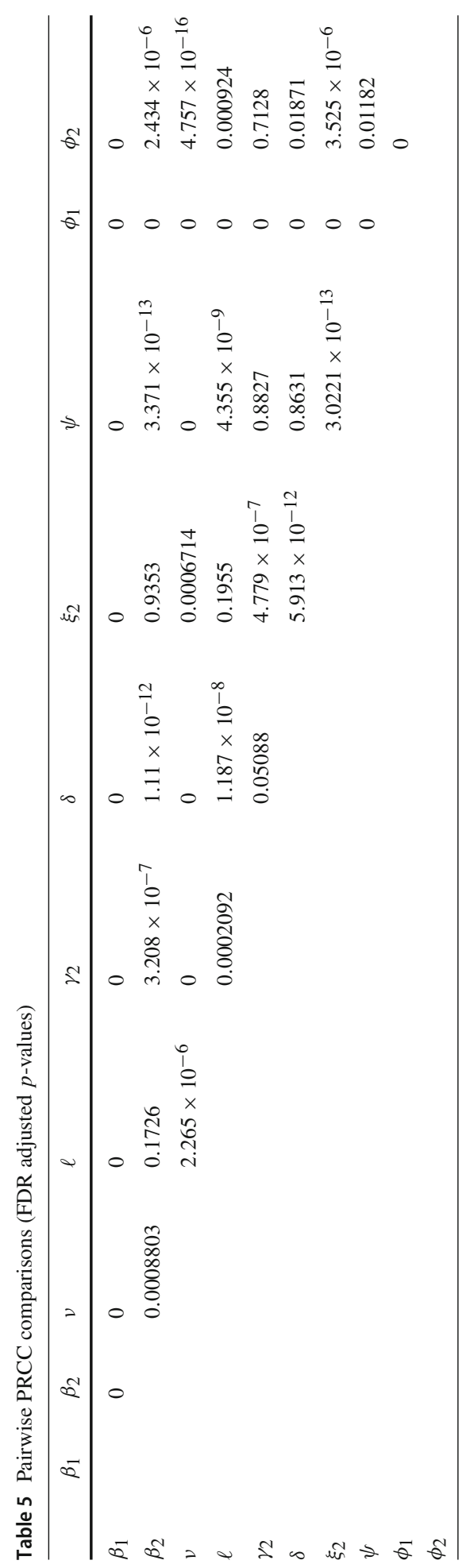


Table 6 Are the parameters different after FDR adjustment?

\begin{tabular}{|c|c|c|c|c|c|c|c|c|c|}
\hline$\beta_{1}$ & $\beta_{2}$ & $v$ & $\ell$ & $\gamma_{2}$ & $\delta$ & $\xi_{2}$ & $\psi$ & $\phi_{1}$ & $\phi_{2}$ \\
\hline$\beta_{1}$ & TRUE & TRUE & TRUE & TRUE & TRUE & TRUE & TRUE & TRUE & TRUE \\
\hline$\beta_{2}$ & & TRUE & FALSE & TRUE & TRUE & FALSE & TRUE & TRUE & TRUE \\
\hline$v$ & & & TRUE & TRUE & TRUE & TRUE & TRUE & TRUE & TRUE \\
\hline$\ell$ & & & & TRUE & TRUE & FALSE & TRUE & TRUE & TRUE \\
\hline$\gamma_{2}$ & & & & & FALSE & TRUE & TRUE & TRUE & FALSE \\
\hline$\delta$ & & & & & & TRUE & FALSE & TRUE & TRUE \\
\hline$\xi_{2}$ & & & & & & & TRUE & TRUE & TRUE \\
\hline$\psi$ & & & & & & & & TRUE & TRUE \\
\hline$\phi_{1}$ & & & & & & & & & TRUE \\
\hline$\phi_{2}$ & & & & & & & & & \\
\hline
\end{tabular}

[29]. In such situations if cases of the disease do occur, they are often easier to isolate or contain.

The proportion of the population that need to be infected to arrive at the herd immunity level depends on the value of the reproduction number, $\mathcal{R}_{0}$, of the disease dynamics and is given by

$$
\frac{\mathcal{R}_{0}-1}{\mathcal{R}_{0}}=1-\frac{1}{\mathcal{R}_{0}} .
$$

Therefore, many of the would-be possible transmissions of the disease fall on those recovered (and hence immune) individuals in the population [6]. That means, after $\left(1-1 / \mathcal{R}_{0}\right) \%$ of the total population is infected the disease may eventually die out (at least temporarily) until the mix of the population changes. For instance, if the disease is with $\mathcal{R}_{0}=2.5$, herd immunity occurs nominally when $(1-1 / 2.5)=60 \%$ of the total population have been infected. If the value of $\mathcal{R}_{0}$ of the disease is large, then herd immunity threshold will be small.

In particular, our parameter estimation from the daily new cases and death data of South Africa results in the value of $\mathcal{R}_{0}$ to be approximately equal to 2.8 . Therefore, according to the above formula, the phenomena of herd immunity for COVID-19 in South Africa can be observed only after nearly $64 \%$ of the total population is recovered from the disease.

Reducing contacts and the transmission probability of the disease as well as isolating/quarantining the infectious individuals are known to have an effect on the actual value of $\mathcal{R}_{0}$. In addition to this, vaccination also contributes to the reduction of this threshold value. The next section considers the implementation of various forms of preventions, as well as isolating/quarantining (and/or hospitalization) of the infectious individuals.

\section{Effect of various disease control measures}

\subsection{Environmental control}

The environment is assumed to be one source that contributes to the spread of the disease. Reducing the effective contact rate of the population to the contaminated environment by disinfecting the environment and applying strict hygiene rules may contribute to the reduction of the new infections. Such interventions have two fold effect; (1) it reduces the environmental 


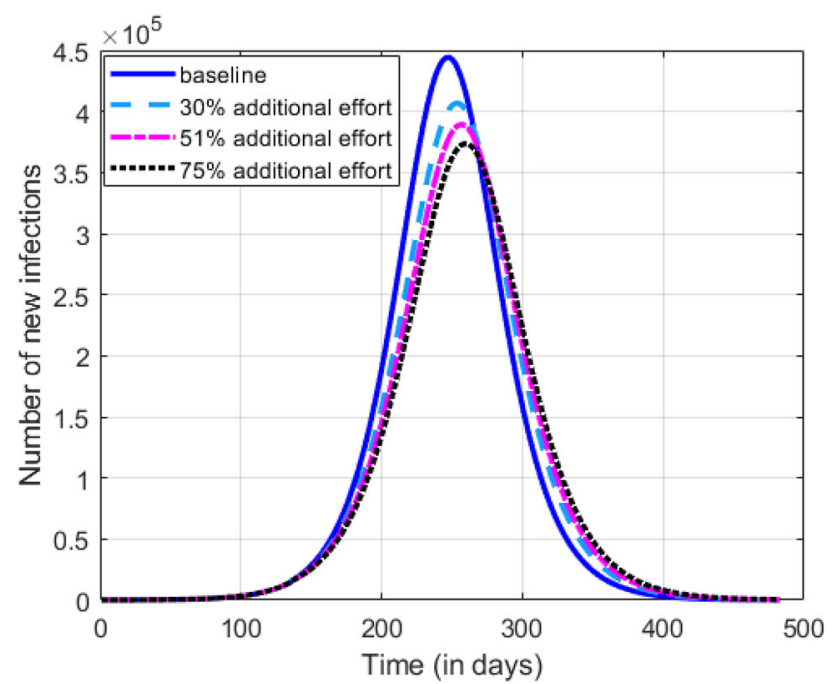

Fig. 4 Effect of environmental control on the number of new infections

transmission rate $\left(\beta_{2}\right)$ and $(2)$ it increases the pathogen decay rate $(\psi)$ when the contaminated surfaces are disinfected. The effect of increasing the percentage of environmental control mechanisms is simulated in Fig. 4. The baseline in the legends of the simulation figures refers to the case where parameter values are the nominal values in Table 2, which portray the dynamics of the disease by the time the data is collected.

We note that improved sanitation and disinfecting of surfaces contribute to not only in lowering the peak values of daily new cases but also the peaks are attained at a later stage. The delayed outbreak may be vital for the government to have enough time to prepare the healthcare system to be able to manage the surge in the number of cases. In addition, the scenarios explored predict that the disease may remain in the population for a relatively long time, as can be seen in Fig. 4 unless other control measures are taken concurrently.

\subsection{Effect of reducing transmission rates}

When people use face masks and keep their distance from others, they are effectively reducing the rate of contact with a potentially infectious individuals. The model can measure the effect of this control measure by the amount of reduction in the transmission rate from infected humans $\left(\beta_{1}\right)$ and from contaminated environment $\left(\beta_{2}\right)$.

The controls are assumed to have an impact on both $\beta_{1}$ and $\beta_{2}$ and the reductions on these values are formulated by multiplicative terms:

$$
1-\varrho_{H} \times \chi_{H} \text { and } 1-\varrho_{E} \times \chi_{E},
$$

where $\varrho_{H}$ and $\varrho_{E}$ represent the efficacy of the control measures on the transmission from humans and environment, respectively, and $\chi_{H}, \chi_{E}$ are representing the percentage of coverage in each case. However, the coverage level may not be constant as the level of alertness of individuals in implementing and enforcing the intervention measures decays as time goes on. To address this, we modeled this values as

$$
\chi_{H}(t)=c_{H} \times e^{a-r t} \text { and } \chi_{E}(t)=c_{E} \times e^{a-r t},
$$



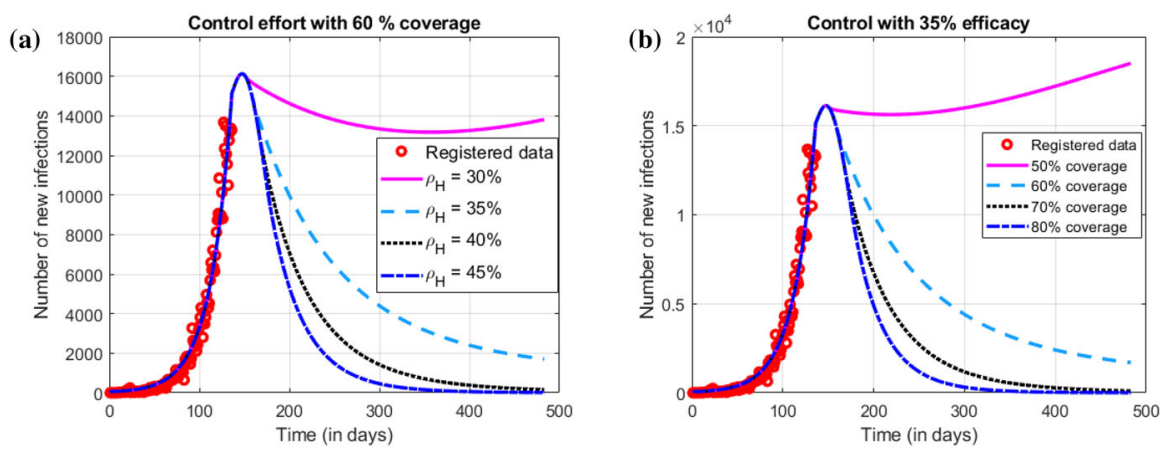

Fig. 5 Effect of the combination of use of face covering and physical distancing on the number of new infections

where $c_{H}$ and $c_{E}$ are the targeted percentages, $a$ is the initial level of alertness and $r$ is the decay rate. In our simulations, we used $a=0.15$ and $r=0.001$ per day.

Therefore, to produce Fig. 5 we used

$$
\left(1-\varrho_{H} \times \chi_{H}(t)\right) \beta_{1} \text { and }\left(1-\varrho_{E} \times \chi_{E}(t)\right) \beta_{2}
$$

in place of $\beta_{1}$ and $\beta_{2}$, respectively, in system equation (28). In the simulation for the current and the next subsections, the implementation of control measures is assumed to start after July 18, which is equivalent to 137 days after the first case was reported in South Africa.

We note on one hand that, if the efficacy of the control measures for both person-to-person transmission and from contaminated surfaces is capped at 35\%, it would require a population coverage of at least $80 \%$ in order to curb new infections or reduce them to minuscule levels. On the other hand, if resources only allow for the coverage of the control effort to only reach up to $60 \%$ of the population, then the intervention processes ought to have the efficacy level of at least $45 \%$ if the new infections are to be reduced to manageable numbers.

\subsection{Effect of increasing random testing and isolating}

The purpose of random testing is to identify asymptomatically infectious individuals and isolate them. Therefore, its main epidemiological effect can be tested by increasing the value of $\phi_{1}$ in the model system (28). The first case in this regard corresponds to the case where there is no other control mechanism except isolating/quarantining the positively tested individuals. The baseline value of $\phi_{1}$ in the simulations corresponds to the nominal values obtained from the data fit and is reported in Table 2. To examine the effect of increasing random testing and isolating/quarantining, we vary the parameter $\phi_{1}$ by several folds from the baseline value and ascertain the potential effect on the peak values of new infections as well as the relative time within which the peaks are attained. Our results are presented in Fig. 6.

As can be observed from Fig. 6, increasing the value of $\phi_{1}$ significantly reduces the new infection with high impact. The numbers in the legend of the figure correspond to the increase in $\phi_{1}$ by 3.8 folds, 5.7 folds and 7.6 folds respectively. Our results show that increasing the detection and isolating of asymptomatic individuals by 3.8 folds leads to a reduction in daily new infections by $64.7 \%$. A 5.7 folds increase in testing and isolating is associated with a $72.8 \%$ reduction in the number of daily new cases. More still, a 7.6 folds increase in testing and isolating/quarantining can lead to up-to $75.7 \%$ reduction in daily new infections. For the 


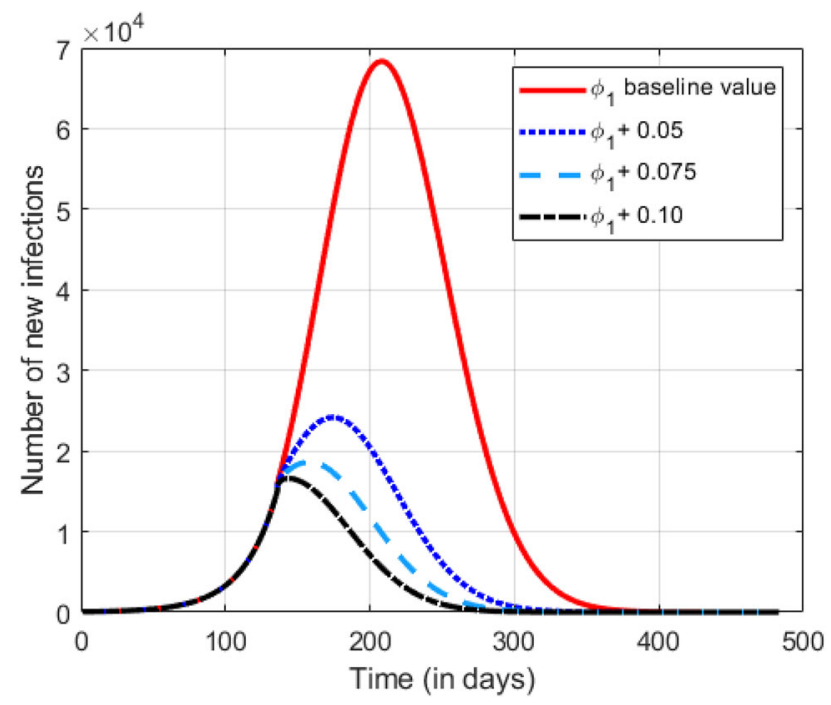

Fig. 6 Impact of scaling up random testing on the number of new infections

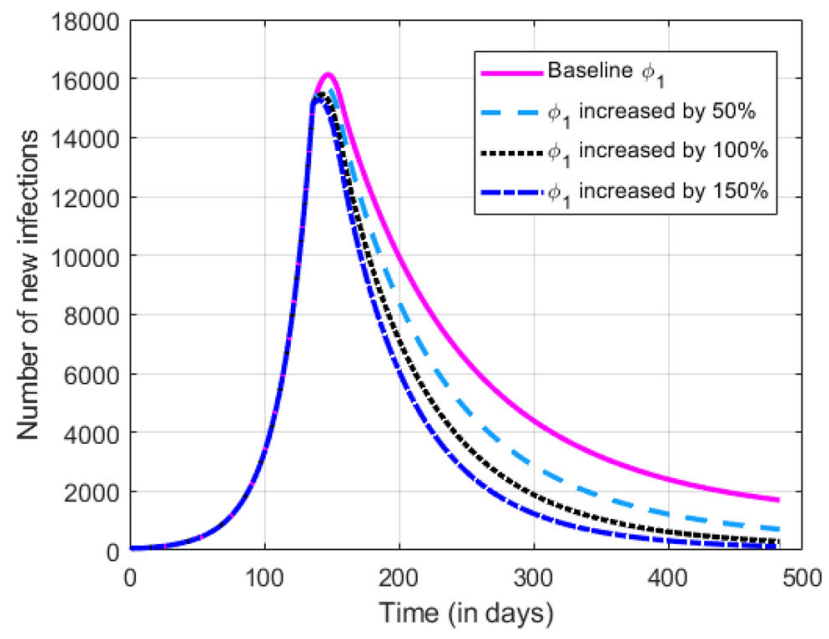

Fig. 7 Impact of scaling up random testing as an additional control.

three cases, the peak is reached earlier by 33 days, 48 days and 66 days respectively. See Fig. 6 for detailed illustration.

On the other hand, while using face covering and physical distancing control measures that have an average of $35 \%$ efficacy with $60 \%$ coverage, it is also possible to increase effectiveness of the controls by increasing the percentage of detecting the asymptomatically infectious individuals in the society. This effect can be seen in Fig. 7. 


\section{Model with hypothetical vaccination and inflow of population}

The baseline model considered in the previous sections assumes that the population is in a closed system and there is no inflow of individuals into the country. This assumption holds only for a short period of time in many countries. However, as can be observed in various places, countries are being opening their borders allowing people to enter from other places, even if some control measures are being implemented to avoid imported infection cases. Such control mechanisms can be implemented strictly if all incoming individuals are using only the legal border crossing of the country. However, in some countries people may cross the borders using informal crossings where authorities may not be able to control them. Such "illegal" ways of crossing borders are common in many countries in Southern Africa as some people have their relatives or their tribal elders residing across borders. This situation is incorporated in model system Eq. (29). In this model, we assume that the rate of inflow (or immigration) is $\Lambda$, of which $q$ percent enter the country through official border crossings so that they can be screened and tested for their status of COVID-19 infection. The remaining proportion are assumed to cross the border illegally and mix with the domestic population freely without them being tested and their disease status known. Here, we also assume that $p$ percent of the illegally crossing individuals are already exposed and the remaining $(1-q)$ proportion of them are asymptomatically infectious.

$$
\begin{aligned}
\dot{M} & =q \Lambda-\left(\alpha_{1}+\alpha_{2}+\mu\right) M, \\
\dot{S} & =\pi+(1-r) \alpha_{1} M-\left(\lambda+\sigma e+\varepsilon_{1}+\mu\right) S, \\
\dot{S}_{e} & =r \alpha_{1} M+\sigma e S-\left[(1-\rho) \lambda+\varepsilon_{2}+\mu\right] S e, \\
\dot{V} & =\varepsilon_{1} S+\varepsilon_{2} S_{e}-\mu V, \\
\dot{E} & =(1-q) p \Lambda+\lambda S+(1-\rho) \lambda S_{e}-(\eta+\mu) E, \\
\dot{A} & =(1-q)(1-p) \Lambda+\ell \eta E-\left(\theta+\phi_{1}+\gamma_{1}+\mu\right) A, \\
\dot{I} & =(1-\ell) \eta E+\theta A-\left(\phi_{2}+\gamma_{2}+\kappa_{1}+\mu\right) I, \\
\dot{Q} & =\alpha_{2} M+\phi_{1} A+\phi_{2} I-\left(\gamma_{3}+\kappa_{2}+\mu\right) Q, \\
\dot{H} & =\kappa_{1} I+\kappa_{2} Q-\left(\gamma_{4}+\mu+\delta\right) H, \\
\dot{R} & =\gamma_{1} A+\gamma_{2} I+\gamma_{3} Q+\gamma_{4} H-\mu R, \\
\dot{W} & =\xi_{1} A+\xi_{2} I-\psi W,
\end{aligned}
$$

Moreover, model system (29) assumes that there is a working vaccination for COVID-19 and the susceptible $(S)$ class of the population are immunized at the rate of $\varepsilon_{1}$ while the aware-susceptible $\left(S_{e}\right)$ class of individuals get vaccinated at the rate of $\varepsilon_{2}$. Due to their high level of awareness and decision to apply any preventive mechanisms, we assume that a larger proportion of individuals from the $S_{e}$ class will be vaccinated as compared to the $S$ class. Therefore, we assume in this model that $\varepsilon_{2}>\varepsilon_{1}$. In addition, hospitalized individuals are considered as a separate class and is denoted by $H$. Therefore, in this case, the force of infection in the transmission dynamics of the disease takes the form

$$
\lambda=\beta_{1} \frac{I+v_{1} A+v_{2} H}{N-Q-M}+\beta_{2} \frac{W}{K+W},
$$

where $N=M+V+S+S_{e}+E+I+A+Q+H+R$. Since the individuals in the $H$ class stay in the protected area being visited by health workers only, their possibility of 
Table 7 Description of the additional state variables and parameters

\begin{tabular}{ll}
\hline Variables & \multicolumn{1}{c}{$\begin{array}{c}\text { Description } \\
\mathrm{M}\end{array}$} \\
$\mathrm{H}$ & \multicolumn{1}{c}{$\begin{array}{c}\text { Newly immigrated population who are isolated for testing } \\
\text { Vaccinated individuals } \\
\text { Hospitalized individuals }\end{array}$} \\
\hline Parameters & Description \\
\hline$\Lambda$ & Rate of immigration to the country \\
$q$ & Rate of legal migration to the population \\
$p$ & Fraction of illegally migrated individuals who are exposed \\
$\varepsilon_{i}$ & Rate of vaccination \\
$r$ & Fraction of immigrated individuals who are tested negative and \\
& who move into the $S_{e}$ class \\
$\alpha_{1}$ & Proportion of immigrated individuals who are tested negative \\
$\alpha_{2}$ & Proportion of immigrated individuals who are tested positive $\left(\alpha_{2}=1-\alpha_{1}\right)$ \\
$\kappa_{i}$ & Rate of hospitalization \\
\hline
\end{tabular}

passing the disease to others is limited. However, since there is a possibility of the disease being transmitted to the people working in the health facilities, we assumed that $v_{2}<<1$.

The mathematical analysis of the model system (29) follows a similar structure as in the analysis presented in Sect. 2. However, we omitted presenting it here for brevity. We rather present the simulation of this model for some varying cases of the newly added parameters and variables.

The description of the additional state variables and new parameters that are involved in the formation of the general model system (29) are presented in Table 7.

\subsection{Impact of immigration}

We consider three cases as follows: (1) In the first case, it is assumed that there is no immigration or emigration, where borders are completely closed. We assume that in the case where there is any cross border movement of goods, "relay driving" is adopted such that trucks are exchanged at the border points and the persons drive only within their respective countries of residence at the time. In this scenario (see blue curve (or the broken line curve) in Fig. 8), the simulations predict an early out break with the new daily infections reaching a peak early on in the course of the pandemic with the peak values registered daily new case at about 14,000. (2) In the second case, we consider a scenario where there is a cross-border movement with about $95 \%$ of the individuals go through legitimate channels while about $5 \%$ of the immigrants are presumed to use illegal crossings and that these individuals are either exposed or asymptomatically infected. This scenario is depicted in the magenta curve (or the solid line curve) in Fig. 8. In this case one can observe a delay in the outbreak but with higher peak values of daily new cases compared to the case with no migration. The model predicted delay in the outbreak can be attributed to a reduced probability of infection since the incidence term is based on standard incidence. Therefore, the influx of many susceptible individuals means that when there are few infected cases at the beginning, the likelihood that susceptible individuals will get in contact with an infected individual will be low. 


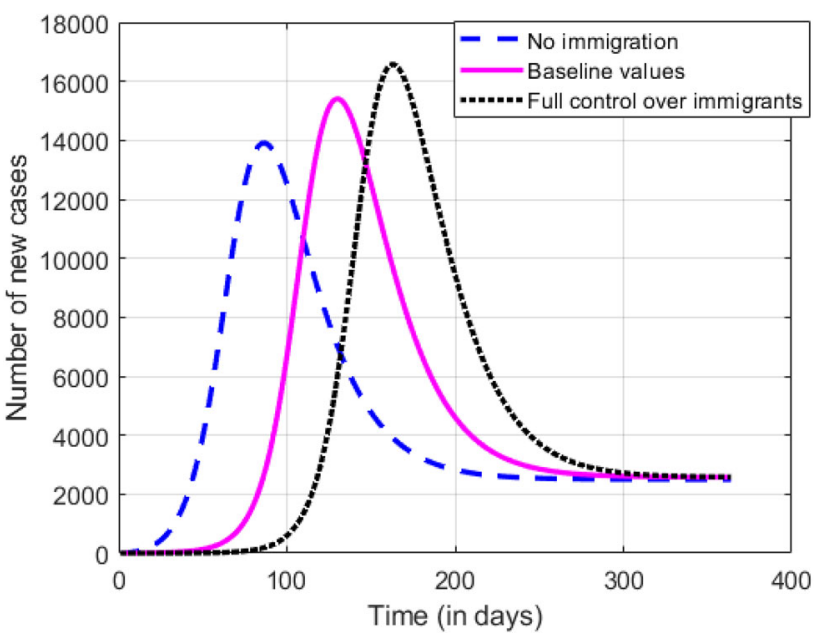

Fig. 8 Impact of controlled and uncontrolled migration on the side of the epidemic. Here, the baseline curve corresponds to the parameter values: $\Lambda=198.37, q=0.95, p=0.66, v_{2}=0.12, r=0.194, \alpha_{1}=$ $0.359, \kappa_{1}=0.25, \kappa_{2}=0.005$ with no vaccination

(3) In the third case, it is assumed that all border posts are in full control of the migration process and standard operating procedures are followed. In this scenario, we assume that at the border posts, only uninfected persons are allowed to cross whereas the infected ones are either turned back or sent to mandatory isolation/quarantine. The predicted simulation for this scenario is depicted in the black curve (or the dotted line curve) in Fig. 8. More still, this inflow of individuals results in a low probability of susceptible persons meeting infected individuals in the initial stages of the pandemic which is depicted by a delayed outbreak. We note however that the peak values reached of daily new infections will be higher. In all the three cases, the equilibrium daily new case remain at relatively the same level and the disease is predicted to remain prevalent with intervention measured implemented.

\subsection{Potential impact of the vaccine}

We incorporate the vaccinated class in the model to establish how the use of a vaccine can potentially influence the number of daily new infections in the long term. We assume availability of a highly effective vaccine and ascertain how vaccination coverage may influence the pandemic dynamics. In the model simulations, we test four vaccination cases as indicated in Fig. 9. The depicted cases include, the baseline case (magenta or the solid line curve depicts a scenario with no vaccination implemented) and cases with $10 \%$ vaccination coverage (blue curve or the broken line curve), 30\% vaccination coverage (red curve or the broken dotted curve), and $98 \%$ vaccination coverage (black curve or the dotted line curve). It is evident that if a highly effective vaccine is used with high coverage, it can potentially curtail new infections.

We also note however that, when immigration is allowed into the country, even a $98 \%$ vaccination coverage may not completely stop new infections. This can be observed from the simulation in Fig. 9 that after a hypothetical effective vaccination is implemented with a coverage of $98 \%$ the number of new cases stabilizes at around 300 new cases per day, which is close to the assumed rate of inflow of immigrants into the country. That means, unless a 


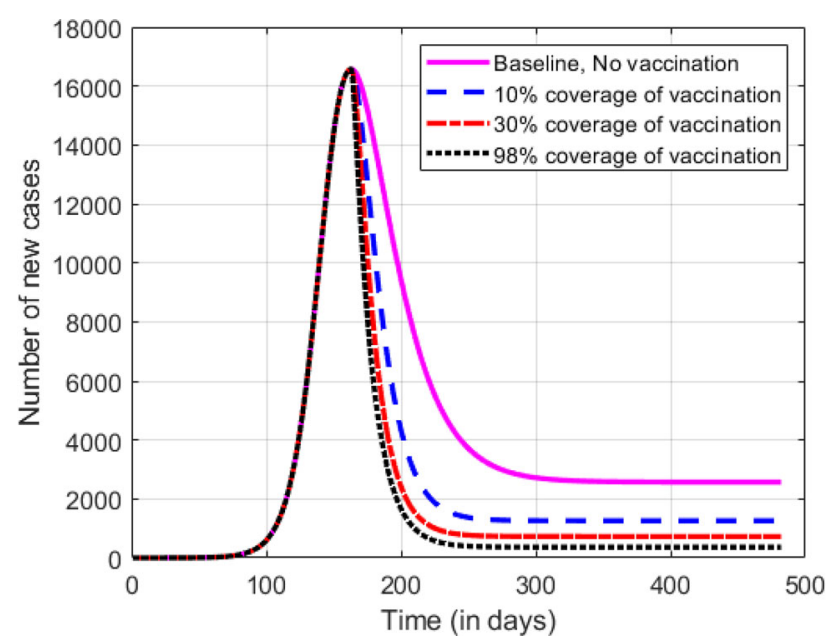

Fig. 9 Impact of applying vaccination on the severity of the infection. The baseline in the figure also corresponds to the parameter values given in the caption for Fig. 8

country is completely isolated from the remaining world, or apply a mandatory vaccination for the incoming individuals, it is not possible to eradicate the infection from the country. We therefore, recommend that, if a vaccine is available, universal vaccination will be the ideal protocol if the new infections are to be curbed.

\section{Discussion and conclusion}

A mathematical model that analyses the transmission dynamics of COVID-19 in presences of both person-to-person contact and environmental transmission and contamination is considered. The model analyses the impact of implementing specific levels of NPI's on the disease severity, the number of daily new cases and conditions under which the preventive measures can be used to effectively contain the disease. The constructed models are composed of the baseline model, and extensions to the baseline model to account for testing and isolating/quarantining/hospitalised of both asymptomatic and symptomatic infected individuals, and a further extension on the model to include the inflow of immigrants to the country as well as a hypothetical vaccination.

The basic analysis of the model properties was done on the baseline model. The model properties including, positivity, boundedness, existence and stability of the equilibrium points were established. The model was shown to have a disease free equilibrium which is globally asymptotically stable and an endemic equilibrium which exists when $\mathcal{R}_{0}$ is greater than unity.

The extended model incorporating testing and isolating/quarantining the confirmed ones was fitted to COVID-19 new cases data for South Africa and the parameter values are determined. For the obtained baseline parameter values, the basic reproduction number $\left(\mathcal{R}_{0}\right)$ evaluated was approximately 2.8. The model was observed to fit well to the data on daily new cases and the daily new deaths. The basic simulation of the baseline model without implementing any control measures predicted that the number of daily new cases would reach the peak around 250 days from the time the first new case was reported. This time period was estimated to be after the first week of November 2020 if no intervention measures were taken. 
Sensitivity analysis of the model was carried out using the Latin hypercube sampling scheme with the output being the reproduction number which is the disease threshold parameter. Our results indicated that the processes described by parameters that are related to contact between susceptible individuals and those infected as well as with the contaminated surfaces had the greatest potential of making the epidemic worse when left unabated. In addition, the relative contribution to new infections by asymptomatic individuals was observed to be highly significant. We further observed that testing and isolating/quarantining infected individuals is significant in curtailing daily new infections. It is therefore, vital to enhance detection and isolating/quarantining of infected persons if the disease is to be contained. Pairwise comparison of the parameter values with significant PRCCs was carried and most pairs of parameters were observed to be significantly different and hence the processes described by such parameters.

Various interventions and control measures including, environmental transmission controls, reduction in transmission rates as well as increasing random testing and isolating/quarantining of infected individuals are studied and numerically tested. Our results show that environmental control and disinfected surfaces is associated with lower and delayed peaks of new daily infections. It was further shown that use of face covering and physical distancing are vital preventive measures in curtailing the number of daily new infected cases. Furthermore, our simulations suggest that, in absence of other control measures, it would require increasing the effort of testing and isolating/quarantining from the baseline value about 7.6 folds to realise a reduction in the number of daily new cases by $75.7 \%$.

Using the extended model that incorporates the use of a hypothetical vaccine and cross border migration, different aspects of vaccination coverage were explored and the potential effect on the daily new infections. In addition, migration patterns were explored considering two scenarios namely; (1) migrations characterised by control by immigration officials implementing SOPs but also in presence of illegal migrations, with just about $5 \%$ of the illegal immigrants either exposed or asymptomatically infected; (2) a case where there is full control of the border crossings with no possibility for illegal crossings. The case characterised with migration through illegal crossings was tested with a consideration of vaccination to ascertain the effect on daily new cases. Our results suggest that in presence of the vaccine, as long as migration is in place, even a $98 \%$ vaccination coverage may not be enough to curb new infections. We therefore suggest that if a highly effective vaccine is made available, it may be necessary to implement universal coverage if the new infections are to be completely contained.

Acknowledgements The authors S.M.K. and H.J.B.N. gratefully acknowledge Botswana International University of Science and Technology (BIUST) that supported their research through the project entitled "Research Initiation grant of the office of the DVCRI of BIUST", with grant number DVC/RDI/2/1/161(34).

\section{References}

1. Blower, S.M., Dowlatabadi, H.: Sensitivity and uncertainty analysis of complex models of disease transmission: an HIV model as an example. Int. Stat. Rev. 62, 229-243 (1994)

2. Busenberg, S., Cooke, K.: Vertically Transmitted Disease: Models and Dynamics, vol. 23. SpringerVerlag, Berlin Heidelberg (1993)

3. Castilo-Chavez, C., Song, B.: Dynamical models of tuberculosis and their application. Math. Biosci. Eng. 1(2), 361-404 (2004)

4. CDC: Interim Clinical Guidance for Management of Patients with Confirmed Coronavirus Disease (COVID-19). Accessed 18 July 2020

5. CDC: Duration of Isolation and Precautions for Adults with COVID-19. Accessed on 18 July 2020 
6. Fine, P., Eames, K., Heymann, D.L.: Herd immunity: a rough gude. Clin. Inf. Dis. 52(7), 911-916 (2011)

7. Gantmacher, F.R.: The Theory of Matrices. Chelsea Publishing Company, New York (1959)

8. Garba, S.M., Lubuma, J.M.S., Tsanou, B.: Modeling the transmission dynamics of the COVID-19 Pandemic in South Africa. Math. Biosci. 328, 108441 (2020)

9. Gumel, A..B., Ruan, S., Day, T., Watmough, J., Brauer, F., den Driessche, P.V., Gabrielson, D., Bowman, C., Alexander, M.E., Ardal, S., Wu, J., Sahai, B.M.: Modelling strategies for controlling SARS outbreaks. Proc. Roy. Soc. Lond. B Biol. Sci. 271(1554), 2223-2232 (2004)

10. Hoare, A., Regan, D.G., Wilson, D.P.: Sampling and statistical analyses tools (SaSAT) for computational modelling. Theor. Biol. Med. Model. 5(4), (2008)

11. Horve, P.F., Lloyd, 569, S., Mhuireach, G.A., Dietz, L., Fretz, M., MacCrone, G., Van Den Wymelenberg, K., Ishaq, S.L.: Building upon current knowledge and techniques of indoor microbiology to construct the next era of theory into microorganisms, health, and the built environment. J. Expo. Sci. Environ. Epidemiol. 30, 219-217 (2020)

12. Kampf, G., Todt, D., Pfaender, S., Steinmann, E.: Persistence of coronaviruses on inanimate surfaces and its inactivation with biocidal agents. J. Hosp. Infect. 104, 246-251 (2020)

13. Kassa, S.M., Ouhinou, A.: Epidemiological models with prevalence dependent endogenous self-protection measure. Math. Biosci. 229, 41-49 (2011)

14. Kassa, S.M., Ouhinou, A.: The impact of self-protective measures in the optimal interventions for controlling infectious diseases of human population. J. Math. Biol. 70, 213-236 (2015)

15. Kassa, S.M., Workineh, Y.H.: Effect of negligence and length of time delay in spontaneous behavioural changes for the response to epidemics. Math. Meth. Appl. Sci. 41, 8613-8635 (2018)

16. Kassa, S., Njagarah, J., Terefe, Y.A.: Analysis of the mitigation strategies for COVID-19: from mathematical modelling perspective. Chaos Solitons Fract. 138, 109968 (2020)

17. LaSalle, J.P.: The stability of dynamical systems. In: CBMS-NSF Regional Conference Series in Applied Mathematics, vol. 25. SIAM, Philadelphia (1976)

18. Lipsitch, M., Allen, J.: Coronavirus reality check: 7 myths about social distancing, busted. USA Today (2020)

19. Long, Q.X., Tang, X.J., Shi, Q.L., Li, Q., Yuan, H.J.D.J., Hu, J.L., et al.: Clinical and immunological assessment of asymptomatic SARS-CoV-2 infections. Nat. Med. 26, 1200-1204 (2020)

20. Macrotrends: South Africa Life Expectancy 1950-2020. Accessed on 18 July 2020

21. Mayo Clinic: Coronavirus Disease 2019 (COVID-19) (2020)

22. Mbewa, D. O.: South Africa Reports First Local Transmission of Coronavirus (2020)

23. Mukandavire, Z., Nyabadza, F., Malunguza, N.J., Cuadros, D.F., Shiri, T., Musuka, G.: Quantifying early COVID-19 outbreak transmission in South Africa and exploring vaccine efficacy scenarios. PLoS One 15(7), (2020)

24. Mushayabasa, S., Ngarakana-Gwasira, E.T., Mushanyu, J.: On the role of governmental action and individual reaction on COVID-19 dynamics in South Africa: a mathematical modelling study. Inf. Med. Unlocked 20, (2020)

25. Ndaïrou, F., Area, I., Nieto, J.J., Torres, D.F.M.: Mathematical modeling of COVID-19 transmission dynamics with a case study of Wuhan. Chaos Solitons Fract. 135, (2020)

26. NICD: First Case of COVID-19 Coronavirus Reported in SA (2020)

27. Ong, S.W.X., Tan, Y.K., Chia, P.Y., Lee, T.H., Ng, O.T., Wong, M.S.Y., Marimuthu, K.: Air, surface environmental, and personal protective equipment contamination by severe acute respiratory syndrome coronavirus 2 (SARS-CoV-2) from a symptomatic patient. JAMA 323(16), 1610-1612 (2020)

28. Poon, L., Peiris, M.: Emergence of a novel human coronavirus threatening human health. Nat. Med. 26, 317-319 (2020)

29. Randolph, H.E., Barreiro, L.B.: Herd immunity: understanding covid-19. Immunity 52, 737-741 (2020)

30. Rothe, C., Schunk, M., Sothmann, P., Bretzel, G., Froeschl, G., Wallrauch, C., Zimmer, T., et al.: Transmission of 2019-nCoV infection from an asymptomatic contact in Germany. N. Engl. J. Med. 382, 970-971 (2020)

31. Stephens, B., Azimi, P., Thoemmes, M.S., Heidarinejad, M., Allen, J.G., Gilbert, J.A.: Microbial exchange via fomites and implications for human health. Curr. Pollut. Rep. 5, 198-213 (2019)

32. Stuart, A.M., Humphries, A.R.: Dynamical Systems and Numerical Analysis. Cambridge University Press, Cambridge (1998)

33. Vandegrift, R., Fahimipour, A.K., Muscarella, M., Bateman, A.C., Van Den Wymelenberg, K., Bohannan, B.: Moving microbes: the dynamics of transient microbial residence on human skin. bioRxiv (2019)

34. van Doremalen, N., Bushmaker, T., Morris, D., Holbrook, M., Gamble, A., Williamson, B., Tamin, A., Harcourt, J., Thornburg, N., Gerber, S., Lloyd-Smith, J., de Wit, E., Munster, V.: Aerosol and surface stability of SARS-CoV-2 as compared with SARS-CoV-1. N. Engl. J. Med. 382, 1564-1567 (2020) 
35. van den Driessche, P., Watmough, J.: Reproduction numbers and sub-threshold endemic equilibria for compartmental models of disease transmission. Math. Biosci. 180, 29-48 (2002)

36. WHO: Coronavirus disease (COVID-2019) Situation Reports. Accessed 8 April 2020

37. Worldometers: COVID-19 Coronavirus Pandemic. Accessed 9 April 2020

38. Worldometers: Coronavirus Incubation Period. Accessed 18 July 2020

39. Xiao, F., Tang, M., Zheng, X., Liu, Y., Li, X., Shan, H.: Evidence for gastrointestinal infection of SARSCoV-2. Gastroenterology 158(6), 1831-1833.e3 (2020)

Publisher's Note Springer Nature remains neutral with regard to jurisdictional claims in published maps and institutional affiliations. 